Bull. Austral. Math. Soc.

37F10, 30D05, 37F50

VoL. $66(2002) \quad[1-8]$

\title{
SOME PROPERTIES OF FATOU AND JULIA SETS OF TRANSCENDENTAL MEROMORPHIC FUNCTIONS
}

\author{
Zheng Jian-Hua, Wang Sheng and Huang Zhi-Gang
}

The radial distribution of Julia sets and non-existence of unbounded Fatou components of transcendental meromorphic functions are investigated in this paper.

\section{Introduction AND Main Results}

Let $f: \mathbf{C} \rightarrow \overline{\mathbf{C}}$ be a transcendental meromorphic function, where $\mathbf{C}$ is the complex plane and $\overline{\mathbf{C}}=\mathbf{C} \cup\{\infty\} . f^{n}(z)$ denotes the $n$-th iterate of $f(z)$, that is, $f^{0}(z)=z, f^{1}(z)$ $=f(z), \ldots, f^{n}(z)=f\left(f^{n-1}(z)\right), n$ is a non-negative integer. $f^{n}(z)$ is well defined for all $z \in \mathbf{C}$, possibly except for an (at most) countable set of poles of $f(z), f^{2}(z), \ldots, f^{n-1}(z)$. Denote by $F_{f}$ the set of those points in $\mathbf{C}$ such that $\left\{f^{n}(z)\right\}_{n=1}^{\infty}$ is well defined and forms a normal family in some neighbourhood of $z . F_{f}$ is called the Fatou set of $f(z)$ and its complement $J_{f}$ the Julia set of $f(z) . F_{f}$ is open and $J_{f}$ is non-empty closed.

Nevanlinna theory is an important tool in the discussion of this paper, some standard notations of which, such as the Nevanlinna deficiency $\delta(\infty, f)$ with respect to $\infty$ and the characteristic function $T(r, f)$ of a meromorphic function $f(z)$ and so on, come mainly from [7]. The lower order $\mu(f)$ of a meromorphic function $f(z)$ is defined as follows:

$$
\mu(f):=\liminf _{r \rightarrow \infty} \frac{\log T(r, f)}{\log r} .
$$

Our first result is about the radial distribution of the Julia sets of transcendental meromorphic functions. In the theory of meromorphic functions, a great deal of work on the relations between the growth in terms of the order and the radial distribution of some value-points of a transcendental meromorphic function were made, for references see $[4,5,10,13]$.

For a $\theta \in[0,2 \pi)$, we say that the Julia set $J_{f}$ has the radial distribution with respect to the radial $\arg z=\theta$, if for any small positive number $\varepsilon>0, \Omega(\theta-\varepsilon, \theta+\varepsilon) \cap J_{f}$ is unbounded, where

$$
\Omega(\theta-\varepsilon, \theta+\varepsilon)=\{z \in \mathbf{C}: \arg z \in(\theta-\varepsilon, \theta+\varepsilon)\} .
$$

Received 31st October, 2001

The work was supported by NSF of China and BRF of Tsinghua University.

Copyright Clearance Centre, Inc. Serial-fee code: 0004-9727/02 \$A2.00+0.00. 
Define

$E:=\left\{\theta \in[0,2 \pi): J_{f}\right.$ has the radial distribution with respect to $\left.\arg z=\theta\right\}$.

It is easy to see that $E$ is closed. By mes $E$ we stands for the linear measure of $E$.

THEOREM 1.1. Let $f(z)$ be a transcendental meromorphic function in $\mathbf{C}$ with $\mu=\mu(f)<\infty$ and $\delta=\delta(\infty, f)>0$. If $\mu=0$, then $E=[0,2 \pi) ;$ If $\mu>0$ and $J_{f}$ has an unbounded component, then

$$
\operatorname{mes} E \geqslant \min \left\{2 \pi, \frac{4}{\mu} \arcsin \sqrt{\frac{\delta}{2}}\right\} \text {. }
$$

We make some remarks on Theorem 1.1.

(1) If $J_{f}$ has only bounded components, we do not know if Theorem 1.1 holds. In this case, $F_{f}$ has at most an unbounded component. If $F_{f}$ has no unbounded components, it is obvious that $E=[0,2 \pi)$. If $F_{f}$ has only an unbounded component $U$, and if $U$ is wandering or periodic of period at least two, then $f$ is bounded in $U$, from the proof of Theorem 1.1 it follows that Theorem 1.1 holds. Then we are left with the case when $U$ is invariant. In this special case, if for some $a \in J_{f}, C_{F_{f}}(a)>0$ (please see the statement before Lemma 2.2 for its definition), then Theorem 1.1 still follows from Lemma 2.2 and the proof of Theorem 1.1.

(2) The condition that $\delta(\infty, f)>0$ is necessary. Observe $f(z)=\lambda \tan z, \lambda \in \Re$, the real axis. It is easy to get $\mu(f)=1$ and $\delta(\infty, f)=0$. It was proved in [3] that when $\lambda>1, J_{f}=\Re$, then $E=\{0, \pi\}$, and mes $E=0$.

When $0<\lambda<1$, the Julia set of $f(z)=\lambda \tan z$ is a Cantor set and the Fatou set consists of one unbounded component, but since $f(z)$ has only two singularity values, it was proved in [11] that for any $a \in J_{f}, C_{F_{f}}(a)>0$.

(3) Baker [2] investigated the radial distribution of the Julia set of a transcendental entire function and constructed an entire function with infinite lower order whose Julia set lies in a horizontal strip. It is well known that an entire function $f$ may only have unbounded simply connnected components of the Fatou set and $\delta(\infty, f)=1$. Therefore, the condition that $f$ has a finite lower order is necessary in Theorem 1.1. A further discussion on this subject of entire functions with finite lower order was made in [9] after Baker [2]. Their methods are not available for the case of meromorphic functions.

Next we consider when mes $E=2 \pi$. If mes $E<2 \pi$, then $F_{f}$ must contain unbounded angle domains. Now [12, Theorem 3$]$ says that $F_{f}$ contains no unbounded angle domains, if for arbitrary positive integer $m$, the following holds

$$
\limsup _{r \rightarrow \infty} \frac{L(r, f)}{r^{m}}=\infty
$$

where $L(r, f):=\min _{|z|=r}\{|f(z)|\}$. Thus we have 
THEOREM 1.2. Let $f(z)$ be a transcendental meromorphic function in $\mathbf{C}$ satisfying (1). Then $E=[0,2 \pi)$.

REMARK. (1) above suggests a further discussion of non-existence of the unbounded periodic components of $F_{f}$, which was investigated in Zheng $[\mathbf{1 1}, \mathbf{1 2}]$.

THEOREM 1.3. Let $f_{j}(j=1,2, \ldots, N)$ be transcendental meromorphic functions. Assume that there exists a sequence $\left\{r_{n}\right\}$ of positive numbers which tends to infinity such that

$$
\lim _{n \rightarrow \infty} \frac{L\left(r_{n}, f_{1}\right)}{r_{n}}=\infty
$$

and for each $j$ and sufficiently large $n$, there is a $R_{j, n} \leqslant r_{n}$, such that

$$
L\left(R_{j, n}, f_{j}\right)>r_{n}, \quad j=2, \ldots, N .
$$

Define $g(z)=f_{1} \circ \cdots \circ f_{N}(z)$. Let $D$ be a hyperbolic domain in C such that for $p>0$, $g^{p}(z): D \rightarrow D$ is analytic. If for some $a \in D, g^{n p}(a) \rightarrow b \in \partial D$, assume, in addition, that $b$ is not an essential singularity point of $g(z)$. Then $D$ is bounded.

We make remarks on Theorem 1.3.

(i) Theorem 1.3 is a generalisation of results in [12]. For example it was proved in [12] that a transcendental meromorphic function has no unbounded (pre)periodic Fatou components if it satisfies (2).

(ii) If $f$ is a transcendental meromorphic function of order $\lambda=\lambda(f)<1 / 2$ and $\delta(\infty, f)>1-\cos \pi \lambda$, then for arbitrarily large $\tilde{r}>0$, we have a $R \leqslant \tilde{r}$ such that $L(R, f)>\tilde{r}$. In fact, we can take $\lambda(f)<\alpha<1 / 2$ such that $\delta(\infty, f)>1-\cos \pi \alpha$. From $[6]$, the set

$$
F:=\left\{r>1: \log L(r, f)>\frac{\pi \alpha}{\sin \pi \alpha}(\cos \pi \alpha+\delta(\infty, f)-1) T(r, f)\right\}
$$

has lower logarithmic density at least $1-(\lambda(f)) / \alpha>0$. Therefore, for all sufficiently large $r>0$, there exists a $R \in\left(r^{1 / d}, r\right)$ such that

$$
L(R, f)>e^{\beta T(R, f)}>R^{d} \geqslant r,
$$

where $\beta=[(\pi \alpha) /(\sin \pi \alpha)](\cos \pi \alpha+\delta(\infty, f)-1)>0$ and $(\alpha / \lambda(f))<d<+\infty$.

This paper was mainly completed by the first author.

\section{Proof of ThEOREM 1.1}

In order to prove the Theorem 1.1, we need the following results. The first result we need is a special version of the main result in [1]. 
LEMMA 2.1. Let $f(z)$ be transcendental and meromorphic in $\mathbf{C}$ with finite positive lower order $\mu=\mu(f)$ and such that $\delta=\delta(\infty, f)>0$. Define for $r>0$

$$
D(r):=\left\{\theta \in[0,2 \pi): \log ^{+}\left|f\left(r e^{i \theta}\right)\right|>\frac{1}{\log r} T(r, f)\right\} .
$$

Then there exists an unbounded sequence $\left\{r_{j}\right\}$ of $r$ such that for sufficiently small $\varepsilon>0$ we have a $j_{0}$ such that when $j \geqslant j_{0}$,

$$
\operatorname{mes} D\left(r_{j}\right) \geqslant \min \left\{2 \pi, \frac{4}{\mu} \arcsin \sqrt{\frac{\delta}{2}}\right\}-\varepsilon .
$$

An open set is called hyperbolic if it has at least three boundary points in $\overline{\mathbf{C}}$. We define the hyperbolic metric on an open set by the hyperbolic metrics of its components. Let $W$ be a hyperbolic open set in $\mathbf{C}$. For an $a \in \mathbf{C} \backslash W$, define

$$
C_{W}(a)=\inf \left\{\lambda_{W}(z)|z-a|: \forall z \in W\right\},
$$

where $\lambda_{W}(z)$ is the hyperbolic density on $W$. It is well-known that $\lambda_{W}(z) \delta_{W}(z) \leqslant 1, z \in$ $W$, where $\delta_{W}(z)$ is the Euclidean distance of $z$ to $\partial W$ and if every component of $W$ is simply connected, then $C_{W}(a) \geqslant 1 / 2$. For $r>0$ and $\theta_{1}, \theta_{2} \in[0,2 \pi), \theta_{1}<\theta_{2}$, define

$$
\Omega\left(r ; \theta_{1}, \theta_{2}\right):=\left\{z: \arg z \in\left(\theta_{1}, \theta_{2}\right),|z|>r\right\} .
$$

LEMMA 2.2. Let $f(z)$ be analytic in $\Omega\left(r_{0} ; \theta_{1}, \theta_{2}\right), U$ a hyperbolic domain and

$$
f: \Omega\left(r_{0} ; \theta_{1}, \theta_{2}\right) \rightarrow U .
$$

If there exists a point $a \in \partial U \backslash\{\infty\}$, such that $C_{U}(a)>0$, then there exists a constant $d>0$ such that for sufficiently small $\varepsilon>0$, we have

$$
|f(z)|=O\left(|z|^{d}\right), z \rightarrow \infty, z \in \Omega\left(r_{0} ; \theta_{1}+\varepsilon, \theta_{2}-\varepsilon\right) .
$$

Proof: Write $\Omega=\Omega\left(r_{0} ; \theta_{1}, \theta_{2}\right)$. Since $f(\Omega) \subset U$, from the Schwarz-Pick Lemma we have

$$
\lambda_{U}(f(z))\left|f^{\prime}(z)\right| \leqslant \lambda_{\Omega}(z), z \in \Omega .
$$

From the definition of $C_{U}(a)$, we have

$$
\lambda_{U}(f(z))\left|f^{\prime}(z)\right| \geqslant C_{U}(a) \frac{\left|f^{\prime}(z)\right|}{|f(z)-a|}, \quad z \in \Omega .
$$

On the other hand, since for $\varepsilon>0$ and $z \in \Omega_{0}=\Omega\left(r_{0} ; \theta_{1}+\varepsilon, \theta_{2}-\varepsilon\right), \delta_{\Omega}(z) \geqslant|z| \sin \varepsilon$, we have

$$
\lambda_{\Omega}(z) \leqslant[|z| \sin \varepsilon]^{-1}, z \in \Omega_{0} .
$$


Combining (7), (8) and (9) gives

$$
c \frac{\left|f^{\prime}(z)\right||d z|}{|f(z)-a|} \leqslant \frac{1}{|z|}|d z|, z \in \Omega_{0},
$$

where $c=C_{U}(a) \sin \varepsilon$. We draw a curve $\gamma$ in $\Omega_{0}$ from a fixed point $b$ to $z$ by connecting $b$ and $|b| e^{i \theta}, \theta=\arg z$ along the circle $\{w:|w|=|b|\}$ and $|b| e^{i \theta}$ and $z$ along the radial $\{w: \arg w=\theta\}$. Then integrating the both sides of (10) along $\gamma$ implies the desired inequality (6).

Proof of Theorem 1.1: If $\mu(f)=0$, from the remark (ii) on Theorem 1.3 we know that $f$ satisfies (1) and therefore from Theorem 1.2 we have $E=[0,2 \pi)$. Now we assume $\mu>0$. For the sake of convenience, put

$$
\sigma=\min \left\{2 \pi, \frac{4}{\mu} \arcsin \sqrt{\frac{\delta}{2}}\right\} .
$$

Now we conversely suppose that mes $E<\sigma$. Take a $t>0$ such that $\sigma-\operatorname{mes} E>t>0$. Since $E$ is closed, $S=[0,2 \pi) \backslash E$ consists of (at most countablely many) open intervals $I$ from which we can then find finitely many open intervals $I_{i}(i=1,2, \ldots, m)$ such that mes $\left(S \backslash \bigcup_{i=1}^{m} I_{i}\right)<K / 2$, where $K=\sigma-\operatorname{mes} E-t>0$. Under the assumption of Theorem 1.1, an application of Lemma 2.1 implies that there exists a sequence $\left\{r_{j}\right\}$ of positive numbers such that mes $D\left(r_{j}\right)>\sigma-t>0$, where $D\left(r_{j}\right)$ is defined as in (4). Obviously

$$
\operatorname{mes}\left(D\left(r_{j}\right) \cap S\right)=\operatorname{mes}\left(D\left(r_{j}\right)\right) \backslash\left(E \cap D\left(r_{j}\right)\right) \geqslant \operatorname{mes} D\left(r_{j}\right)-\operatorname{mes} E \geqslant K>0 .
$$

Thus for each $j$ we have

$$
\begin{aligned}
\operatorname{mes}\left(\left(\bigcup_{i=1}^{m} I_{i}\right) \cap D\left(r_{j}\right)\right) & =\operatorname{mes}\left(S \cap D\left(r_{j}\right)\right)-\operatorname{mes}\left(\left(S \backslash \bigcup_{i=1}^{m} I_{i}\right) \cap D\left(r_{j}\right)\right) \\
& >K-\frac{K}{2}=\frac{K}{2},
\end{aligned}
$$

so there exists an open interval $I=I_{i_{0}} \subset S$ such that for infinitely many $j$,

$$
\operatorname{mes}\left(D\left(r_{j}\right) \cap I\right)>\frac{K}{2 m}>0 .
$$

It is easy to see that we can assume that for each $j,(11)$ holds. Write $I=(\alpha, \beta)$. Take a positive number $\varepsilon$ such that

$$
\operatorname{mes}\left(D\left(r_{j}\right) \cap I_{2 \varepsilon}\right)>\frac{K}{3 m}>0(j=1,2, \ldots),
$$

where we denote by $I_{d}$ the interval $(\alpha+d, \beta-d)$ for $0<2 d<\beta-\alpha$. It is easy to see from $I \cap E=\emptyset$ that there exists a positive $R$ such that $\Omega\left(R ; I_{\epsilon}\right)=\{z \in C$ : $|z| \geqslant R$ and $\left.\arg z \in I_{\varepsilon}\right\} \subset F_{f}$. Now by applying Lemma 2.2 to $f$ in $\Omega\left(R ; I_{\varepsilon}\right)$, we have

$$
|f(z)| \leqslant C_{0}|z|^{p}, z \in \Omega\left(R ; I_{2 \varepsilon}\right),
$$


where $C_{0}$ and $p$ are both positive constants. Then

$$
\cdot \int_{I_{2 \varepsilon} \cap D\left(r_{j}\right)} \log ^{+}\left|f\left(r_{j} e^{i \theta}\right)\right| d \theta \leqslant \int_{I} p \log r_{j} d \theta+O(1)=O\left(\log r_{j}\right) .
$$

On the other hand, applying Lemma 2.1 to $f$ gives

$$
\int_{I_{2 \varepsilon} \cap D\left(r_{j}\right)} \log ^{+}\left|f\left(r_{j} e^{i \theta}\right)\right| d \theta \geqslant \operatorname{mes}\left(I_{2 \varepsilon} \cap D\left(r_{j}\right)\right) \frac{T\left(r_{j}, f\right)}{\log r_{j}}>\frac{K}{3 m} \frac{T\left(r_{j}, f\right)}{\log r_{j}} .
$$

Combining (14) with (15) gives

$$
T\left(r_{j}, f\right)=O\left(\left(\log r_{j}\right)^{2}\right) .
$$

Then $\mu(f)=0$. We get a contradiction.

Theorem 1.1 follows.

\section{Proof of TheOREM 1.3}

In order to prove Theorem 1.3, we need the following result.

Lemma 3.1. Let $D$ be a domain with at least three boundary points and $f$ be meromorphic in $\mathbf{C}$ except possibly at most countably many essential sigularity points such that $f(D) \subset D$. Then one of the following mutually exclusive possibilities can occur.

(1) There is a subsequence of $\left\{f^{n}(z)\right\}$ which converges to $z$ in $D$.

(2) $f^{n}(z) \rightarrow b \in \bar{D}, \bar{D}$ is the closure of $D$.

Lemma 3.1 can be proved from the arguments of Heins [8]. We also need the following result, which is due to Zheng [12] and of independent significance.

LEMMA 3.2. Let $U$ be an unbounded hyperbolic domain and $f: U \rightarrow U$ analytic. If

$$
\left.f^{n}(z)\right|_{U} \rightarrow \infty, \quad n \rightarrow \infty,
$$

then there exists a curve $\gamma$ tending to infinity and a constant $\mathcal{L}>1$, such that $f(\gamma) \subset \gamma$ and

$$
\frac{|z|}{\mathcal{L}} \leqslant|f(z)| \leqslant \mathcal{L}|z|, \forall z \in \gamma .
$$

Proof of Theorem 1.3: Suppose that $D$ is unbounded. Take a point $a \in D$ and a positive number $M$ such that

$$
|a|<M \text { and }\left|f_{j} \circ \ldots \circ f_{N}\left(g^{i}(a)\right)\right|<M, \text { for } j=1,2, \ldots, N \text { and } i=0,1, \ldots, p \text {. }
$$

From (2) and (3) for arbitrarily large $K>3$ we can take a sufficiently large $R>M$ such that

$$
L\left(R, f_{1}\right)>K R
$$


and for each $j$ there exists a $M \leqslant R_{j} \leqslant R$ such that

$$
L\left(R_{j}, f_{j}\right)>R .
$$

Now we draw in D a curve $\gamma$ connecting $a$ and a point in $\{|z|=R\}$ such that $\gamma \subset\{|z|$ $\leqslant R\}$. It is easy to see that $\left\{|z|=R_{N}\right\} \cap \gamma \neq \emptyset$ and from (16), $f_{N}(\gamma) \cap\{|z|<M\} \neq \emptyset$, and then from (18) when $j=N$ we have

$$
f_{N}(\gamma) \cap\{|z|=R\} \neq \emptyset .
$$

Obviously from (16), $f_{N-1} \circ f_{N}(\gamma) \cap\{|z|<M\} \neq \emptyset$ and from (19), $f_{N}(\gamma) \cap\{|z|$ $\left.=R_{N-1}\right\} \neq \emptyset$. Again applying (18) when $j=N-1$ gives that $f_{N-1} \circ f_{N}(\gamma) \cap\{|z|=R\}$ $\neq \emptyset$. Inductively we get

$$
f_{2} \circ \cdots \circ f_{N-1} \circ f_{N}(\gamma) \cap\{|z|=R\} \neq \emptyset .
$$

Then there exists a point $z_{1} \in \gamma$ such that $\left|f_{2} \circ \cdots \circ f_{N-1} \circ f_{N}\left(z_{1}\right)\right|=R$, and from (17) we have $\left|g\left(z_{1}\right)\right|>K R$. This implies that $g(\gamma) \cap\{|z|=R\} \neq \emptyset$. Take a segment of $g(\gamma)$, denoted by $\gamma_{\mathbf{1}}$, from $g(a)$ to $\{|z|=R\}$ such that $\gamma_{1} \subset\{|z| \leqslant R\}$. Repeating the above process to $\gamma_{1}$ gives that $g\left(\gamma_{1}\right) \cap\{|z|=R\} \neq \emptyset$, so $g^{2}(\gamma) \cap\{|z|=R\} \neq \emptyset$. Inductively we obtain $g^{p-1}(\gamma) \cap\{|z|=R\} \neq \emptyset$. Now take a curve $\gamma_{p-1}$ from $g^{p-1}(\gamma)$ connecting $g^{p-1}(a)$ and a point in $\{|z|=R\}$ such that $\gamma_{p-1} \subset\{|z| \leqslant R\}$. As we did for $\gamma$ in the first step, we have (20) for $\gamma_{p-1}$, so that we have (20) for $g^{p-1}(\gamma)$. Then there exists a point $z^{*} \in \gamma$ such that $\left|f_{2} \circ \cdots \circ f_{N-1} \circ f_{N}\left(g^{p-1}\left(z^{*}\right)\right)\right|=R$, and from (17) we have $\left|g^{p}\left(z^{*}\right)\right|>K R$.

If $\left\{g^{n}(a)\right\}$ is unbounded, then for some $0 \leqslant m \leqslant p-1,\left.g^{n p+m}\right|_{D} \rightarrow \infty(n \rightarrow \infty)$. It is easy to see from unboundedness of $D$ and (2) and (3) that $g^{m}(D)$ is also unbounded. Since $g^{p}\left(g^{m}(D)\right) \subset g^{m}(D)$, applying Lemma 3.2 to $g^{p}$ in $g^{m}(D)$ gives the existence of $\Gamma$ tending to $\infty$ from $g^{m}(a)$ in $g^{m}(D)$ such that $\left|g^{p}(z)\right| \leqslant L|z|, z \in \Gamma$, where $L$ is a positive constant. In the above discussion, we replace $\gamma$ by a segment curve of $\Gamma$ in $\{|z| \leqslant R\}, D$ by $g^{m}(D)$ and $K$ by $2 L$, then we have a point $z^{*} \in \Gamma$ such that $\left|g^{p}\left(z^{*}\right)\right|>2 L R$. On the other hand, $\left|g^{p}\left(z^{*}\right)\right| \leqslant L\left|z^{*}\right| \leqslant L R$, so that $L R \geqslant 2 L R$. We get a contradiction. Then $\left\{g^{n}(a)\right\}$ is bounded.

If $\left\{\left.g^{n}\right|_{D}\right\}$ has only constant limit functions, then from Lemma $3.1 g^{n p+m}(a)$ $\rightarrow p_{m}(n \rightarrow \infty), 0 \leqslant m \leqslant p-1$. If $p_{0} \in D$, then $p_{m} \in g^{m}(D), 0 \leqslant m \leqslant p-1$ so that we can reqiure that (16) also holds for $i>p$. If $p_{0} \in \partial D$, we can also do this under the assumption of Theorem 1.3 about this case. Thus it is clear that $g^{n p}(\gamma)$ cannot tend to $p_{0}$. A contradiction we get gives that $\left\{\left.g^{n}\right|_{D}\right\}$ has a non-constant limit function. Then all the limit points of $\left\{g^{n}(a)\right\}$ must be analytic points of $f_{j} \circ \cdots \circ f_{N}(z),(j=1,2, \ldots, p)$ so that we can make (16) hold for $i>p$. Applying Lemma 3.1 implies that there is a subsequence $\left\{g^{n_{k} p}\right\}$ of $\left\{g^{n p}\right\}$ such that

$$
g^{n_{k} p}(z) \rightarrow z,(k \rightarrow \infty)
$$


in $D$. When $n_{k}$ is sufficiently large, we have

$$
\left|g^{n_{k} p}(z)\right|<(K-1)|z|, z \in \gamma
$$

On the other hand, from (21) and the discussion in the first paragraph, there is a $z_{n_{k}} \in \gamma$ such that

$$
K R<\left|g^{n_{k} p}\left(z_{n_{k}}\right)\right|<(K-1)\left|z_{n_{k}}\right| \leqslant(K-1) R .
$$

This is impossible.

Theorem 1.3 follows.

\section{REFERENCES}

[1] A. Baernstein, 'Proof of Edrei's spread conjecture', Proc. London Math. Soc. 26 (1973), 418-434.

[2] I.N. Baker, 'Sets of non-normality in iteration theory', J. London Math. Soc. 40 (1965), 499-502.

[3] W. Bergweiler, 'Iteration of meromorphic functions', Bull. Amer. Math. Soc. 29 (1993), 151-188.

[4] A. Edrei, 'Meromorphic functions with three radially distributed values', Trans. Amer. Math. Soc. 78 (1955), 276-293.

[5] A. Edrei and W.H.J. Fuchs, 'Meromorphic functions with several deficient values', Trans. Amer. Math. Soc. 93 (1959), 292-328.

[6] A.A. Gol'dberg and O.P. Sokolovskaya, 'Some relations for meromorphic functions of order or lower order less than one', Izv. Vyssh. Uchebn. Zaved. Mat. 31 (1987), 26-31. Translation: Soviet Math. (Iz. VUZ) 31 (1987), 29-35.

[7] W.K. Hayman, Meromorphic functions, Oxford Mathematical Monographs (Clarendon Press, Oxford, 1964).

[8] M.H. Heins, 'On the iteration of functions which are analytic and single-valued in a given multiply-connected region', Amer. J. Math. 63 (1957), 461-480.

[9] J. Qiao, 'Stable domains in the iteration of entire functions', (Chinese), Acta Math. Sinica 37 (1994), 702-708.

[10] G.H. Zhang, Theory of entire and meromorphic functions, (Chinese) (Science Press Sinica, 1986).

[11] J.H. Zheng, 'Unbounded domains of normality of entire functions of small growth', Math. Proc. Cambridge Philos. Soc. 128 (2000), 355-361.

[12] J.H. Zheng, 'On non-existence of unbounded domains of normality of meromorphic functions', J. Math. Anal. Appl. 264 (2001), 479-494.

[13] J.H. Zheng, 'On the growth of meromorphic functions with two radially distributed values', J. Math. Anal. Appl. 206 (1997), 140-154.

Department of Mathematical Sciences

Tsinghua University

Beijing 100084

People's Republic of China

e-mail: jzheng@math.tsinghua.edu.cn 